\title{
Fibrilación auricular de primera vez en evento vascular cerebral isquémico en el servicio de urgencias
}

\author{
Undiagnosed atrial fibrillation in patients with an ischemic cerebral vascular event
}

\begin{abstract}
Franklin Ríos-Jaimes ${ }^{1 *}$, Olivia A. Otero-González², Enrique Villarreal-Ríos ${ }^{3}$ y M. Carlota García-Gutiérrez ${ }^{4}$
${ }^{1}$ Servicio de Urgencias, Hospital General Regional N. ${ }^{0}$ 1, Instituto Mexicano del Seguro Social, Querétaro de Arteaga, Qro.; ${ }^{2}$ Servicio de Urgencias, Hospital General de Zona N. ${ }^{\circ}$ 3, Instituto Mexicano del Seguro Social, Ciudad Mante, Tamps.; ${ }^{3}$ Unidad de Investigación Epidemiológica y en Servicios de Salud Querétaro, Instituto Mexicano del Seguro Social, Querétaro de Arteaga, Qro.; ${ }^{4}$ Facultad de Medicina, Universidad Autónoma de Querétaro, Qro. México
\end{abstract}

\section{Resumen}

Objetivo: Determinar la incidencia de fibrilación auricular no diagnosticada en pacientes con evento vascular cerebral tipo isquémico. Métodos: Diseño transversal descriptivo en pacientes mayores de 18 años con diagnóstico de evento vascular cerebral de tipo isquémico, atendidos en el servicio de urgencias. El tamaño de la muestra lo integraron el total de expedientes clínicos de pacientes con diagnóstico de evento vascular cerebral isquémico $(n=199)$. Se incluyeron todos los pacientes a los que se les realizó electrocardiograma. Se estudió edad, sexo, antecedente de diabetes mellitus y antecedente de hipertensión arterial. El evento vascular cerebral se diagnosticó con tomografía axial computarizada avalada por médico radiólogo; se consideró fibrilación auricular cuando en el electrocardiograma se identificó onda $R-R$ irregular, ausencia de onda $P$ y actividad auricular irregular; la lectura e interpretación fue realizada por el médico urgenciólogo. El análisis estadístico incluyó porcentajes, intervalos de confianza para porcentajes y cálculo de probabilidad de ocurrencia de evento binomial. Resultados: El promedio de edad de los pacientes con evento vascular cerebral es 72.37 años (IC 95\%: 70.91-73.82), predomina el sexo masculino con un 57.7\% (intervalo de confianza del 95\% [IC 95\%]: 50.8-64.7). El diagnóstico de hipertensión arterial está presente en el 74.8\% (IC 95\%: 68.8-80.9) de los pacientes. La incidencia de fibrilación auricular de primera es el 72.36\% (IC 95\%: 66.0-78.6). Conclusión: En la población estudiada la incidencia de fibrilación auricular no diagnosticada previamente en pacientes con evento vascular cerebral isquémico es alta.

Palabras clave: Fibrilación auricular. Evento vascular isquémico. Incidencia.

\begin{abstract}
Objective: To determine the incidence of undiagnosed atrial fibrillation in patients with an ischemic cerebral vascular event. Methods: A descriptive cross-sectional design was carried out in patients older than 18 years with a diagnosis of ischemic cerebral vascular event, attended in the emergency department. The sample size was made up of the total clinical records of patients with a diagnosis of ischemic cerebral vascular event $(n=199)$. All patients who underwent electrocardiogram were included. Age and sex were studied; history of diabetes mellitus and history of hypertension. The cerebral vascular event was
\end{abstract}

Correspondencia:

${ }^{\star}$ Franklin Ríos-Jaimes

E-mail: franklinro85@gmail.com
Fecha de recepción: 23-07-2020

Fecha de aceptación: 09-12-2020 DOI: 10.24875/ACM.20000354
Disponible en internet: 18-02-2021 Arch Cardiol Mex. 2021;91(4):453-457 www.archivoscardiologia.com

1405-9940 / @ 2020 Instituto Nacional de Cardiología Ignacio Chávez. Publicado por Permanyer. Este es un artículo open access bajo la licencia CC BY-NC-ND (http://creativecommons.org/licenses/by-nc-nd/4.0/). 
diagnosed with a computerized axial tomography endorsed by a radiologist; atrial fibrillation was considered when an irregular $R-R$ wave, absence of $P$ wave, and irregular atrial activity were identified on the electrocardiogram. The reading and interpretation was performed by the emergency physician. Statistical analysis included percentages, confidence intervals for percentages and calculation of probability of occurrence of binomial event. Results: The average age of the patients with a cerebral vascular event is 72.37 years (95\% Cl: 70.91-73.82), the male sex predominates with $57.7 \%$ (95\% Cl: $50.8-64.7)$. The diagnosis of arterial hypertension is present in $74.8 \%$ (95\% Cl: $68.8-80.9)$ of the patients. The incidence of first-time atrial fibrillation is $72.36 \%$ (95\% Cl: 66.0-78.6). Conclusion: In the studied population, the incidence of previously undiagnosed atrial fibrillation in patients with ischemic cerebrovascular event is high.

Key words: Atrial fibrillation. Ischemic cerebral vascular event. Incidence.

\section{Introducción}

La enfermedad vascular cerebral no es un problema de salud menor: la tasa de mortalidad se ha reportado en 28.8 por 100,000 habitantes ${ }^{1}$, es motivo de dependencia en adultos (de 1,393 a 3,864 por 100,000 personas) ${ }^{2}$ y el costo en atención y productividad supera los 4.6 billones de euros ${ }^{3,4}$.

La incidencia del evento vascular cerebral isquémico se ha incrementado, condición explicada por el aumento en la esperanza de vida y la aparición de comorbilidades, incluidas las de origen cardiaco $^{1,5}$, específicamente la fibrilación auricular, patología propia del adulto en torno a la cual se pronostica incremento de la incidencia condicionada por factores de riesgo que incluyen edad, hipertensión y obesidad ${ }^{6}$.

Al respecto se han descrito las modificaciones eléctricas asociadas al envejecimiento que determinan alteraciones tisulares y celulares de las aurículas (fibrosis, apoptosis, hipertrofia, desorganización de las gap junction y acortamiento del potencial de acción) ${ }^{7}$. De igual forma, la hipertensión arterial genera hipertrofia ventricular izquierda, condicionando disfunción diastólica con alteración en el llenado de la cavidad, dilatación auricular ipsilateral y retraso en la velocidad de conducción, favoreciendo la presencia de fibrilación auricular ${ }^{8,9}$, y con ella flujos turbulentos que propician trastornos de la coagulación, condición óptima para desarrollar procesos tromboembólicos ${ }^{10-13}$. Se manifiesta electrocardiográficamente con irregularidad de la onda $\mathrm{R}-\mathrm{R}$, ausencia de onda P y actividad auricular irregular ${ }^{14,15}$.

La incidencia de fibrilación auricular en el evento vascular cerebral isquémico fluctúa entre el 20.0 y el $43.2 \%{ }^{16-18}$, valor que desciende al $10 \%$ cuando la fibrilación auricular no ha sido diagnosticada previamente ${ }^{19,20}$. Las variaciones pueden explicarse por el avance del conocimiento médico en torno a esta arritmia y al desarrollo de las técnicas de monitoreo cardiaco ${ }^{21}$.

Realizar el diagnóstico temprano de la fibrilación auricular implica el inicio de la terapia anticoagulante efectiva, condición que puede constituirse como acción preventiva del accidente vascular cerebral isquémico y todas sus complicaciones ${ }^{22}$.

En este contexto, el objetivo fue determinar la incidencia de fibrilación auricular no diagnosticada en pacientes con evento vascular cerebral tipo isquémico.

\section{Metodología}

Diseño transversal descriptivo en expedientes clínicos de pacientes mayores de 18 años con diagnóstico de evento vascular cerebral de tipo isquémico, atendidos del 1 de enero de 2017 al 1 de enero del 2019 en el servicio de urgencias de un hospital general regional perteneciente a una institución de seguridad social.

El evento vascular cerebral isquémico fue diagnosticado clínicamente con la presencia de focalización neurológica súbita sin otra causa que la de origen vascular ${ }^{23}$, confirmado con tomografía axial computarizada certificada por médico radiólogo. Fueron incluidos todos los pacientes con reporte de electrocardiograma y se eliminaron los pacientes con datos clínicos de evento vascular cerebral en los cuales no se pudo establecer el diagnóstico en el estudio de imagen.

Se estudió edad, sexo, antecedente de diabetes mellitus y antecedente de hipertensión arterial. El diagnóstico de fibrilación auricular se estableció con la presencia electrocardiográfica de onda R-R irregular, ausencia de onda $\mathrm{P}$ y actividad auricular irregular ${ }^{24}$; la lectura e interpretación del electrocardiograma fue realizada por el médico urgenciólogo.

Se identificaron las características de la población estudiada y se estimó la incidencia de fibrilación auricular no diagnosticada. Posteriormente se identificaron las características de la población con evento cerebral vascular isquémico y fibrilación auricular.

El tamaño de muestra se calculó con la fórmula de porcentajes para población finita, considerando que el total de eventos vascular cerebral isquémico en el periodo de estudio fue 199 ( $n=199)$, la estimación se 
Tabla 1. Prevalencia de comorbilidades en la población estudiada

\begin{tabular}{|l|c|c|c|}
\hline Comorbilidad & Prevalencia & \multicolumn{2}{|c|}{ IC 95\% } \\
\cline { 2 - 4 } & & Inferior & Superior \\
\hline Hipertensión arterial con o sin diabetes mellitus & 74.8 & 68.8 & 80.9 \\
\hline O diabetes o hipertensión arterial & 56.7 & 49.8 & 63.7 \\
\hline Diabetes mellitus con o sin hipertensión arterial & 50.2 & 43.2 & 57.2 \\
\hline Solo hipertensión arterial & 40.7 & 33.9 & 40.7 \\
\hline Diabetes mellitus e hipertensión arterial juntas & 34.1 & 27.5 & 40.8 \\
\hline Solo diabetes mellitus & 16.0 & 10.9 & 21.1 \\
\hline Ni diabetes mellitus, ni hipertensión arterial & 9.0 & 5.0 & 13.0 \\
\hline
\end{tabular}

IC $95 \%$ : intervalo de confianza del $95 \%$.

realizó con nivel de confianza del 95\% ( $Z$ alfa $=1.96$ ) para dos zonas de rechazo, con base en la hipótesis que identifica la incidencia de fibrilación auricular en el evento vascular cerebral en el $43.2 \%{ }^{25}(p=0.432)$ y margen de error del $5 \%(d=0.05)$. El tamaño calculado fue 130.48 , no obstante, se incluyeron el total de los casos, para un total de muestra de 199.

El análisis estadístico incluyó promedios, porcentajes, intervalos de confianza para promedios, intervalos de confianza para porcentajes y cálculo de probabilidad de ocurrencia del evento en una distribución binomial $\left({ }_{n} C_{x} p^{x} q^{n-x}\right)$.

El proyecto fue autorizado por el Comité Local de Investigación y el Comité de Ética en Investigación. Posterior a ello se solicitó autorización de la dirección médica del hospital para ingresar al expediente clínico de los pacientes con diagnóstico de evento vascular cerebral isquémico, información que permitió construir la base de datos.

\section{Resultados}

Inicialmente se incluyeron 234 pacientes con diagnóstico clínico de evento vascular cerebral isquémico, no obstante, en 35 pacientes la tomografía axial computarizada no confirmó el diagnóstico y se eliminaron del estudio. El total de pacientes estudiados fueron 199.

El promedio de edad de los pacientes con evento vascular cerebral fue 72.37 años (intervalo de confianza del 95\% [IC 95\%]: 70.91-73.82), predominó el sexo masculino con 57.7\% (IC 95\%: 50.8-64.7).

El diagnóstico de hipertensión arterial estuvo presente en el 74.8\% (IC 95\%: 68.8-80.9) de los pacientes. En la tabla 1 se presenta la prevalencia de la diabetes y de la diabetes asociada con hipertensión.
Tabla 2. Cálculo de probabilidad de tener antecedente de fibrilación auricular no diagnosticada en cinco pacientes atendidos en urgencias por evento vascular cerebral isquémico

\begin{tabular}{|l|l|}
\hline $\begin{array}{l}\text { Pacientes con antecedente de fibrilación } \\
\text { auricular no diagnosticada }\end{array}$ & Probabilidad \\
\hline 0 & 0.0016 \\
\hline 1 & 0.0211 \\
\hline 2 & 0.1106 \\
\hline 3 & 0.2894 \\
\hline 4 & 0.3789 \\
\hline 5 & 0.1984 \\
\hline
\end{tabular}

La incidencia de fibrilación auricular no diagnosticada previamente fue del 72.36\% (IC 95\%: 66.0-78.6).

En un escenario supuesto, si llegan al servicio de urgencia cinco pacientes con evento vascular cerebral isquémico la probabilidad de que exactamente cuatro tengan fibrilación auricular no diagnosticada es del $37.89 \%$. En la tabla 2 se presenta el resto de las probabilidades.

En la población con evento vascular cerebral isquémico sin antecedente previo de fibrilación auricular, pero con fibrilación auricular en el momento del evento vascular, el promedio de edad fue 72.10 años (IC 95\%: 70.34-73.85), y predominó el sexo masculino, con un 54.8\% (IC 95\%: 46.6-63.0).

En esta población la comorbilidad más prevalente fue hipertensión arterial, con un 74.3\% (IC 95\%: 67.081.5). En la tabla 3 se presenta el resto de las comorbilidades. 
Tabla 3. Prevalencia de comorbilidades en la población con evento vascular cerebral isquémico

\begin{tabular}{|l|c|c|c|}
\hline Comorbilidad & Prevalencia & \multicolumn{2}{|c|}{ IC 95\% } \\
\cline { 2 - 4 } & & Inferior & Superior \\
\hline Hipertensión arterial con o sin diabetes mellitus & 74.3 & 67.0 & 51.5 \\
\hline O diabetes mellitus o hipertensión arterial & 57.6 & 49.4 & 62.4 \\
\hline Diabetes mellitus con o sin hipertensión arterial & 54.1 & 45.9 & 46.8 \\
\hline Solo hipertensión arterial & 38.8 & 30.8 & 43.3 \\
\hline Diabetes mellitus e hipertensión arterial & 35.4 & 27.5 & 25.1 \\
\hline Solo diabetes mellitus & 18.7 & 12.3 & 11.1 \\
\hline Ni diabetes mellitus ni hipertensión arterial & 6.9 & 2.74 & \\
\hline
\end{tabular}

IC $95 \%$ : intervalo de confianza del $95 \%$.

\section{Discusión}

El avance tecnológico y la extensa literatura de los factores de riesgo para fibrilación auricular favorecen el diagnóstico oportuno, no obstante, la incidencia reportada de fibrilación auricular no diagnosticada en pacientes con evento vascular cerebral isquémico no confirma el postulado ${ }^{23}$. Este es un problema de salud que involucra al Médico en general, pero particularmente al que ejerce la práctica clínica en el primer nivel de atención, personaje al que se debe proporcionar la información al respecto.

En este trabajo fueron excluidos los pacientes con isquemia cerebral transitoria, en ellos el diagnóstico se estableció desde la perspectiva clínica y por diferentes médicos, escenario que puede constituirse como sesgo. La inclusión de pacientes con evento vascular cerebral confirmado por tomografía axial computarizada permite tener consistencia en el diagnóstico del evento vascular, no obstante, es una realidad que existen diferentes mecanismos, uno de ellos la fibrilación auricular.

Idealmente el diagnóstico de fibrilación auricular lo debe realizar el cardiólogo, pero es una realidad que la formación académica del médico urgenciólogo lo acredita para ello.

En la literatura los reportes de incidencia de fibrilación auricular oculta o no diagnosticada previamente en pacientes con evento vascular cerebral son variables, los valores fluctúan entre el 10.019,20, 20.0 $0^{16,17} \mathrm{y}$ $25.0 \%{ }^{17}$, cuando la medición se hace en pacientes con diagnóstico previo de fibrilación auricular la incidencia corresponde al $43.2 \%{ }^{18}$. Al comparar estos valores con la incidencia de fibrilación auricular encontrada en la población estudiada la diferencia es evidente; no obstante, por el tipo de diseño empleado no se puede dar una explicación concluyente en torno a las discrepancias, sin embargo se pueden asumir hipótesis, entre ellas la alta prevalencia de factores de riesgo para fibrilación auricular en la población estudiada, específicamente la alta prevalencia de hipertensión y diabetes, así como la edad?.

En este trabajo lo realmente importante no son los factores de riesgo, lo relevante es la ausencia de diagnóstico temprano de fibrilación auricular, escenario que se constituye como una crítica a los servicios de salud, conscientes de que no se investigó si el paciente con fibrilación auricular era usuario de los servicios de salud de primer nivel. Los resultados son una llamada de atención para favorecer el diagnóstico temprano que permita iniciar con terapia específica encaminada a prevenir las complicaciones de la fibrilación auricular, entre ellas el evento vascular cerebral isquémico ${ }^{25,26}$.

El cálculo de probabilidad de ocurrencia de la fibrilación auricular en pacientes atendidos en urgencias por evento vascular cerebral isquémico es muy alto, así lo demuestra la estimación binomial presentada en el trabajo, conocimiento que respalda la importancia del diagnóstico y tratamiento temprano de la fibrilación auricular y la necesidad de establecer una política de salud en torno al tema.

Ante la alta prevalencia de fibrilación auricular en pacientes con evento vascular cerebral isquémico, la detección temprana de fibrilación auricular en pacientes con factores de riesgo debería ser una prioridad en la atención en medicina familiar, escenario que propicia el manejo farmacológico específico y la prevención del evento vascular cerebral isquémico, no obstante no se 
puede asegurar la prevención, esta es una hipótesis; el trabajo que aquí se presenta no puede asegurarlo, para ello se tendría que realizar un estudio de causalidad.

\section{Conclusiones}

En población estudiada la incidencia de fibrilación auricular no diagnosticada previamente en pacientes con evento vascular cerebral isquémico es alta y superior a lo reportado en la literatura.

\section{Financiamiento}

Para la realización de este trabajo no se recibió financiamiento específico.

\section{Conflictos de intereses}

Los autores declaran no tener conflicto de intereses.

\section{Responsabilidades éticas}

Protección de personas y animales. Los autores declaran que para esta investigación no se han realizado experimentos en seres humanos ni en animales.

Confidencialidad de los datos. Los autores declaran que han seguido los protocolos de su centro de trabajo sobre la publicación de datos de pacientes.

Derecho a la privacidad y consentimiento informado. Los autores declaran que en este artículo no aparecen datos de pacientes.

\section{Bibliografía}

1. Chiquete E, Ruiz-Sandoval JL, Murillo-Bonilla LM, Arauz A, Villarreal Careaga J, Barinagarrementería $F$, et al. Mortalidad por enfermedad vascular cerebral en México, 2000-2008: Una exhortación a la acción. Rev Mex Neuroci. 2011;12(5):235-41.

2. Mar J, Sainz-Ezkerra M, Moler-Cuiral JA. Calculation of prevalence estimates through differential equations: application to stroke-related disability. Neuroepidemiology 2008;31(1):57-66.

3. Prince MJ, Wu F, Guo Y, Gutierrez Robledo LM, O'Donnell M, Sullivan R, et al. The burden of disease in older people and implications for health policy and practice. Lancet. 2015;385:549-62.
4. Luengo-Fernández R, Leal J, Gray A, Petersen S, Rayner M. Cost of cardiovascular diseases in the United Kingdom. Heart. 2006;92(10):1384-9.

5. Ruiz-Sandoval JL, Cantú-Brito C, Chiquete E, Parra-Romero G, Arauz A, de Jesús-Villaseñor $T$, et al. Enfermedad vascular cerebral isquémica aguda en mayores de 75 años en la primera década del siglo XXI en México. Rev Mex Neuroci. 2017;18(2):42-53.

6. Candel FJ, Matesanz M, Cogolludo F, Candel I, Mora C, Bescos T, et al. Prevalencia de fibrilación auricular y factores relacionados en una población del centro de Madrid. AN Med Interna. 2004;21(10):477-82.

7. Segule R. ¿Es posible prevenir la fibrilación auricular y sus complicaciones? Rev Med Clin Condes. 2012;23(6):732-41.

8. Halley JS, Connolly SJ. Atrial fibrillation: Hypertension as a causative agent, risk factor for complication, and potential therapeutic target. Am J Cardiol. 2003:91(10A):9-14.

9. Verdecchia P, Dagenais G, Healey J, Gao P, Dans AL, Chazova I, et al. Blood pressure and other determinants of new-onsent atrial fibrillation in patients at high cardiovascular risk in the Ongoing Telmisartan Alone and in Combination with Ramipril Global Endpoint Trial/Telimisartan Randomized Assessment Study in ACE intolerant subjects with cardiovascular disease studies. J Hypertens. 2012;30(5):1004-14.

10. Agewall S, Camm J, Barón Esquivias G, Budts W, Carerj S, Casselman F, et al. Guía ESC 2016 sobre el diagnóstico de la fibrilación auricular desarrollada en colaboración con la EACTS. Rev Esp Cardiol. 2017;70(1):50-84.

11. Andersson T, Magnoson A, Bryngelsson IL, Frøbert O, Henriksson KM, Edvardsson $\mathrm{N}$, et al. All-cause mortality in 272,186 patients hospitalized with incident atrial fibrillation 1995-2008: a Swedish nation wide long-term case-control study. Eur Heart J. 2013;34:1061-7.

12. Shin S, Burnett RT, Kwong JC, Hystad P, van Donkelaar A, Brook JR, et al. Ambient air pollution and the risk of atrial fibrillation and stroke: $A$ population based cohort study. Environ Health Perpect. 2019;127(8):1-15.

13. Cesasuolo JO, Cipriano LE, Sposato LA. The complexity of atrial fibrillation newly diagnosed after schemic stroke and transient ischemic attack: advances and uncertainties. Curr Opin Neurol. 2017;30(1):28-37.

14. January CT, Wann LS, Alpert JS, Calkins H, Cigarroa JE, Cleveland Jr JC, et al. $2014 \mathrm{AHA} / \mathrm{ACC} / \mathrm{HRS}$ Guideline for the management of patients with atrial fibrillation: Executive summary. A report of the American College of Cardiology/American Heart Association Task Force on Practice Guidelines and the Heart Rhythm Society. Circulation. 2014;130(23):2071-104.

15. Fuster V, Rydén LE, Cannom DS, Crijns HJ, Curtis AB, Ellenbogen KA et al. 2011 ACC/AHA/ARS Focused updates incorporated into the ACC/ AHA/ARS 2006, Guidelines for the management of patients with atrial fibrillation: A report of the American College Cardiology Fundation/American Heart Association Task Force on Practice Guidelines. Circulation. 2011;123:e269-317.

16. Cantú-Brito C, Ruiz Sandoval JL, Murillo-Bonilla LM, Chiquete E, León-Jiménez $\mathrm{C}$, Arauz A, et al. Acute care and one-year outcome of Mexican patients with first-ever acute ischaemic stroke: the PREMIER study. Rev Neurol. 2010;51(11):641-9.

17. Moreno Peña LE, Hernández Hervis IT, Moreno Peña R, García Peñate G, Suárez Pozo R. Fibrilación auricular en pacientes con ictus isquémico en Hospital Universitario Comandante Faustino Pérez. 2017. Rev Med Electrón. 2018;40(2):360-70.

18. Rodríguez Duarte S. Práctica diaria. Factores de riesgo cardiovascular en enfermos adultos con evento vascular cerebral. Enf Neurol. 2010;9(2):90-3.

19. Reyes CW. Fibrilación auricular y accidente cerebrovascular. Una arritmia subdiagnosticada y subtratada. Rev Urug Cardiol. 2017:32(2):190-1.

20. Zorrilla JP. Fibrilación auricular oculta en el accidente cerebrovascular isquémico: Buscad y hallaréis. Neurolog Argent. 2011;3(1):74-5.

21. Sposato LA, Riccio PM, Hachinski VH. Poststroke atrial fibrillation: cause or consequence? Critical review of current views. Neurology. 2014;82:1180-6

22. Elizari MV. Consenso de fibrilación auricular. Rev Argent Cardiol. 2005; 73(6):469-85.

23. Arauz A, Ruíz-Franco A. Enfermedad vascular cerebral. Rev Fac Med. 2012;55(3):11-21.

24. Mora-Pabón G. Evaluación de la fibrilación auricular mediante electrocardiograma y Holter. Rev Colomb Cardiol. 2016;23(S5):27-33.

25. Turackhia M, Shafrin J, Bognar K, Trocio J, Abdulsattar Y, Wiederkehr D, et al. Estimated prevalence of undiagnosed atrial fibrillation in the United States. PLoS One. 2018;13(4):1-11.

26. Wolf PA, Abbott RD, Kannel WB. Atrial fibrillations an independent risk factor for stroke: The Framingham study. Stroke. 1991;22(8):983-8. 